\title{
Using Accurate Solid Angle Tools when Comparing EDS Detector Geometries
}

\author{
Patrick Camus ${ }^{1}$ and Reinhard Buchhold ${ }^{1,2}$ \\ ${ }^{1}$ EDAX Inc., A Division of Ametek, Materials Analysis Division, 95 McKee Place, Mahwah, NJ 07430 \\ ${ }^{2}$ Werth Messtechnik, Siemensstrasse 19, Giessen, 35394, Germany
}

Analysts should strive for the EDS system that provides the highest efficiency for their analytical work. For detectors, that typically means starting with the geometry with the best solid angle (SA) value. Manufacturers should be designing interfaces to maximize the solid angle of the detectors without compromising the integrity of the electron columns. Accurate modeling of the solid angle is required for both analysts and manufacturers so that everyone is satisfied with the detector performance. This paper will show that new solid angle calculations must be used for accurate solid angle modeling. Application of these models to new geometries illustrates the performance benefits of creative geometric designs.

Historically, the SA (measured in steradian, sR, or milli-steradians, msR) of a detector was approximated by the simplistic equation $\mathrm{SA}=\mathrm{A} / \mathrm{d}^{2}$ where $\mathrm{A}$ is the active area of the detector and $\mathrm{d}$ is the detector to sample distance. For small detectors at large distances, this approximation is quite good. However, for large area detectors (which are currently quite popular) and for most TEM applications (which use very short values for $\mathrm{d}$ ), this simple equation is not valid. This was recognized $[1,2]$ but little has been done in the reporting of correct values.

Analytic equations for solid angle values have been reported [3, 4], and a web site has been developed [5] to provide users the ability to accurately model and compare the geometries of EDS detectors. Four geometries are present including the typical SEM geometry where the detector axis points to the sample, the typical TEM geometry where the detector axis is perpendicular to the sample axis (horizontal mount), a rare square detector geometry, and an annular detector.

As mentioned earlier, the simple calculation of solid angle is not valid for all geometries but most detectors are reported using these values. New geometries are being developed that need to have the best estimates of the solid angle values and should use these new calculations. A few old geometries and a few new geometries will be analyzed using both the simple and detailed calculations to compare the values.

A typical SEM geometry is examined where $10 \mathrm{~mm}^{2}$ and $30 \mathrm{~mm}^{2}$ detectors are compared. Most designs for these detectors have the same $\mathrm{d}$, for instance $45 \mathrm{~mm}$ in this example.

Using these values, simplistic and analytic SA values can be found.

\begin{tabular}{|c|c|c|c|}
\hline $\mathrm{d}(\mathrm{mm})$ & $\mathrm{A}\left(\mathrm{mm}^{2}\right)$ & Simple SA (msR) & Analytic SA (msR) \\
\hline 45 & 10 & 5 & 5 \\
\hline 45 & 30 & 15 & 15 \\
\hline
\end{tabular}

In this example, both SA methods provide the same values. There is no primary benefit to using the analytic method.

Another SEM example shows a practical application of the SA calculations in comparing potential EDS detector geometries. When comparing detectors and considering SA values, the A is not always the primary consideration for the efficiency because the $d$ term can have a significant effect. This usually occurs when the tubing for the detectors is not the same and the d must increase for a fatter mounting tube to avoid hitting any item with in the SEM chamber, usually the pole piece. This example compares a $60 \mathrm{~mm}^{2}$ in a small diameter tube mounted at $40 \mathrm{~mm}$ and an $80 \mathrm{~mm}^{2}$ detector in a larger diameter tube that must be mounted at a larger $\mathrm{d}$ of $50 \mathrm{~mm}$. 
Using these values, simplistic and calculated SA values can be found.

\begin{tabular}{|c|c|c|c|}
\hline $\mathrm{d}(\mathrm{mm})$ & $\mathrm{A}\left(\mathrm{mm}^{2}\right)$ & Simple SA (msR) & Calculated SA (msR) \\
\hline 40 & 60 & 36 & 36 \\
\hline 50 & 80 & 32 & 31 \\
\hline
\end{tabular}

Firstly, the values provided by the 2 calculation methods are essentially the same (within round-off errors) for both detectors. Intuition would indicate that the larger $80 \mathrm{~mm}^{2}$ detector should provide the greater SA value. However, the increased d actually reduces its SA value to less than that for the smaller $60 \mathrm{~mm}^{2}$ detector.

The next example is for a TEM geometry where $10 \mathrm{~mm} 2$ and $30 \mathrm{~mm} 2$ detectors are compared. The primary differences to the SEM example are that (1) $d=10$ which is significantly shorter than for the SEM geometry, and (2) the axis of the detector does not point to the sample. This geometry is very typical for TEM that have a side entry mount location. In addition, this geometry also needs a take-off angle, for which 20 degrees is used.

Using these values, simplistic and analytic SA values can be found.

\begin{tabular}{|c|c|c|c|}
\hline $\mathrm{d}(\mathrm{mm})$ & $\mathrm{A}(\mathrm{mm} 2)$ & Simple SA (msR) & Analytic SA (msR) \\
\hline 10 & 10 & 94 & 88 \\
\hline 10 & 30 & 282 & 249 \\
\hline
\end{tabular}

In this example, the simple SA method over estimates the true SA values by $7 \%$ and $13 \%$ for the 2 detectors. SA values for TEM geometries should not be reported using the simple method but should always use the more accurate analytic method.

Two further TEM examples compare traditional detector geometries similar to that used above to new very-high SA designs all using $30 \mathrm{~mm}^{2}$ detectors. The primary change in the new designs is the insertion to shorter $\mathrm{d}$ values and the decrease in the take-off angle to fit within the confines of the column opening.

TEM 1

\begin{tabular}{|c|c|c|c|c|}
\hline $\mathrm{d}(\mathrm{mm})$ & $\mathrm{A}(\mathrm{mm} 2)$ & Take-off Angle & Simple SA (msR) & Analytic SA (msR) \\
\hline 11.4 & 30 & 14.77 & 223 & 200 \\
\hline 9.1 & 30 & 9.5 & 357 & 312 \\
\hline
\end{tabular}

TEM 2

\begin{tabular}{|c|c|c|c|c|}
\hline $\mathrm{d}(\mathrm{mm})$ & $\mathrm{A}(\mathrm{mm} 2)$ & Take-off Angle & Simple SA (msR) & Analytic SA (msR) \\
\hline 9.6 & 30 & 20.1 & 306 & 269 \\
\hline 7.9 & 30 & 18.4 & 456 & 388 \\
\hline
\end{tabular}

These results illustrate (1) that the simple calculations are not very accurate and (2) that the optimized geometry detectors should perform much better than traditional geometries.

Solid angle values for EDS detectors are an indication of the x-ray detection efficiency of the system. Analytic equations should be used for the most accurate SA calculations. Comparisons of detector geometry can aid an analyst in selecting the best detector for their application.

[1] N. J. Zaluzec, Micros. Microanal. 15, 2009, 93-98

[2] N. J. Zaluzec, Micros. Microanal. 19 (Suppl 2), 2013, 1262-3

[3] John T. Conway, Nuclear Instr. And Methods in Phys Research A, 614, 2010 17-27

[4] Richard J. Mathar, http://www.mpia.de/ mathar

[5] http://tpm.amc.anl.gov/NJZTools/XEDSSolidAngle.html 